\title{
High-Definition Intravascular Ultrasound Versus Optical Frequency Domain Imaging for the Detection of Calcium Modification and Fracture in Heavily Calcified Coronary Lesion
}

Masaru Ishida ( $\nabla$ maishida@iwate-med.ac.jp)

Iwate Medical University https://orcid.org/0000-0002-5960-7806

Yuya Oshikiri

Iwate Medical University

Takumi Kimura

Iwate Medical University

Ryohei Sakamoto

Nakadori General Hospital

Yudai Shimoda

Iwate Medical University

Yu Ishikawa

Iwate Medical University

Yorihiko Koeda

Iwate Medical University

Yuya Taguchi

Iwate Medical University

Tomonori Itoh

Iwate Medical University

Yoshihiro Morino

Iwate Medical University

\section{Research Article}

Keywords: Calcified lesion, Intravascular ultrasound (IVUS), Optical frequency domain imaging (OFDI), Orbital atherectomy, Rotational atherectomy

Posted Date: November 10th, 2021

DOI: https://doi.org/10.21203/rs.3.rs-1052510/v1 
License: (c) (i) This work is licensed under a Creative Commons Attribution 4.0 International License. Read Full License

Version of Record: A version of this preprint was published at The International Journal of Cardiovascular Imaging on January 6th, 2022. See the published version at https://doi.org/10.1007/s10554-021-025218. 


\section{Abstract \\ Purpose}

While optical frequency domain imaging (OFDI) can delineate calcium modification and fracture, the capability of high-definition intravascular ultrasound (HD-IVUS) for detecting these remains unclear. This study evaluated the diagnostic accuracy of HD-IVUS for assessing calcium modification and fracture as compared to OFDI.

\section{Methods}

HD-IVUS and OFDI were used during orbital or rotational atherectomy procedures conducted for 21 heavily calcified coronary lesions in 19 patients. With OFDI assessment used as the gold standard, diagnostic accuracies of HD-IVUS for calcium modification and fracture were compared every $1 \mathrm{~mm}$ to the matched pre-stenting images $(n=1,129)$. Calcium modification, as assessed by OFDI, was defined as polished and concave-shaped calcium. For HD-IVUS, calcium modification was defined as the presence of reverberation with concave-shaped calcium. In both assessments, the definition of calcium fracture was defined as a slit or complete break in the calcium plate.

\section{Results}

Calcified plaque was found in $86.4 \%$ of analyzed OFDI images. Calcium modification and fracture were detected in $20.6 \%$ and $11.0 \%$ of detected calcified plaques. Sensitivity, specificity, positive and negative predictive values of HD-IVUS detection for calcium modification and fracture were $54.4 \%, 97.8 \%, 86.7 \%$, $89.1 \%$ and $86.0 \%, 94.5 \%, 58.2 \%, 96.8 \%$, respectively. Discordance cases between both assessments demonstrated that heterogeneous calcium visualized by OFDI, separated calcium and guide wire artifact can be misdiagnosed.

\section{Conclusion}

Diagnostic accuracies of HD-IVUS for assessing calcium modification and fracture were modest as compared to OFDI. These results suggest that OFDI guidance is more feasible during treatment of heavily calcified coronary lesions versus HD-IVUS guidance.

\section{Introduction}

Even when using current-generation drug-eluting stents, a calcified coronary lesion remains a risk factor for poor clinical outcome of percutaneous coronary intervention (PCI) [1, 2], which is primarily caused by stent underexpansion due to thick and circumferential coronary calcium [3,4]. Therefore, before implanting a stent in a heavily calcified lesion, the $\mathrm{PCl}$ operator needs to confirm whether or not the target 
lesion is expandable. Optical frequency domain imaging (OFDI) and optical coherence tomography (OCT), which can delineate calcium modification by atherectomy devices and calcium fracture caused by angioplasty, are considered to be an appropriate imaging modality for evaluating the expandability before stent implantation in heavily calcified coronary lesions [5].

Recently, $60 \mathrm{MHz}$ high-definition intravascular ultrasound (HD-IVUS), which has a relatively highresolution image as compared to conventional IVUS, has been widely used to assess the presence of a favorable lesion preparation after atherectomy or ballooning, in addition to helping to confirm stent optimization [6-8]. Since HD-IVUS does not require performing contrast flush during imaging, HD-IVUS can be used to perform OFDI in more difficult situations such as the tight stenotic or ostial lesions and for the patient with renal insufficiency [8]. Although HD-IVUS can be used in many of these difficult situations, the capability of HD-IVUS in detecting calcium modification and fracture has yet to be assessed. To evaluate the diagnostic accuracy of HD-IVUS for heavily calcified lesions, we compared HDIVUS and OFDI with regard to the detection of calcium modification and fracture.

\section{Materials And Methods}

\section{Study design and population}

This observational study evaluated patients with coronary artery disease (CAD) who were admitted to Iwate Medical University Hospital or Nakadori General Hospital from March 2019 to October 2020. This study complies with the guidelines of the Declaration of Helsinki, and the Ethics Committee of each of the participating institutions approved the study protocol (MH2019-125). Patients who underwent orbital atherectomy $(\mathrm{OA})$ or rotational atherectomy (RA) for treatment of heavily calcified coronary lesions and who underwent both HD-IVUS imaging and OFDI during PCI were included in this study. A total of 19 CAD patients with 21 heavily calcified coronary lesions were enrolled in this study that compared evaluations between HD-IVUS and OFDI of the calcium modification and fracture.

\section{$\mathrm{PCl}$ procedure and intravascular image acquisition}

In this study, all of the target lesions included the heavily calcified segment, which was defined by the angiographic presence of radio-opacities noted without cardiac motion prior to contrast injection involving both sides of the arterial wall in at least one location, the total length of the calcified segment was at least $15 \mathrm{~mm}$ and partially extended into the target lesion, or there was a presence of $\geq 270^{\circ}$ of calcium at 1 cross section that was observed via OFDI or HD-IVUS [9]. All PCI procedures were primarily performed under OFDI guidance. Both OFDI and HD-IVUS imaging were performed just before (pre-) stenting and immediately after the PCl. Either the Lunawave ${ }^{\circledR}$ OFDI system with the FastView ${ }^{\circledR}$ imaging catheter (Terumo Corporation, Tokyo, Japan) or the Visicube ${ }^{\circledR} 60 \mathrm{MHz}$ HD-IVUS system with the AltaView ${ }^{\circledR}$ imaging catheter (Terumo Corporation, Tokyo, Japan) were used for image acquisition. The OFDI images were obtained at a rate of $158 \mathrm{frames} / \mathrm{s}$ with a $40 \mathrm{~mm} / \mathrm{s}$ pullback speed (4 images $/ \mathrm{mm}$ ), while the HD-IVUS imaging was obtained at a rate of 90 images/s with a $9 \mathrm{~mm} / \mathrm{s}$ pullback speed (10 
images $/ \mathrm{mm}$ ). To facilitate stent delivery and expansion, OA or RA were performed prior to the first ballooning. The choice of using OA or RA was determined by each physician based on the pre-PCI angiography or intravascular imaging. After performing the pre-stenting intravascular imaging following the plaque modification using cutting, scoring or a non-compliant balloon, the biodegradable-polymer drug-eluting stents were implanted in the culprit lesion. Finally, the post-stent intravascular imaging was performed, with the implanted stents then optimized based on the expert consensus document of the European Association of PCls [6]. Minimum stent area (MSA), either the distal or proximal edge stent dissection, and large stent malapposition were assessed using post-PCl intravascular images. To avoid coronary vasospasm, patients were administered an intracoronary injection of nicorandil, isosorbide dinitrate or nitroglycerin prior to each intravascular imaging.

\section{OFDI and HD-IVUS image analysis}

Using an off-line viewer system, all images were independently assessed by two experienced reviewers (M.I. and T.K.). To perform a head-to-head comparison between both imaging modalities, every $1 \mathrm{~mm}$ of the HD-IVUS images were linked to the OFDI images through the use of landmarks such as branches and calcium appearance. Coronary calcium was defined as a signal-poor or heterogeneous region with a sharply delineated border by OFDI [10]. It was further defined as bright dense echoes with acoustic shadowing of underlying tissue by HD-IVUS [11]. The presence of layered calcium on the OFDI images was defined as having $\geq 1$ layer with different coronary calcium. The calcium nodule assessed by OFDI was defined as an accumulation of nodular calcification (small calcium deposits) exhibiting disruption of the fibrous cap on the calcium plate [12]. The calcium nodule assessed by HD-IVUS was defined as a protruded calcium with a convex or irregular luminal surface [13]. For the analysis of the pre-stenting OFDI images, calcium modification was defined as the presence of polished and concave-shaped calcium [14]. For the analysis of the pre-stenting HD-IVUS images, calcium modification was defined as the presence of reverberation with the presence of concave-shaped calcium [15]. Calcium fracture was defined as a slit or complete break in the calcium plate that was identified in the pre-stenting OFDI or HDIVUS [5] (Figure 1). With regard to the post-stenting OFDI and IVUS findings, reference lumen area, MSA, stent expansion (MSA / reference lumen area), particular findings including major stent edge dissection and major acute stent malapposition were all assessed based on the previously reported consensus document [6]. Notably, major stent edge dissection was defined as $\geq 60$ degrees of the circumference of the vessel at the site of dissection and / or $\geq 2 \mathrm{~mm}$ in length. Major acute stent malapposition was defined as $<0.4 \mathrm{~mm}$ with longitudinal extension $<1 \mathrm{~mm}$. In-stent tissue protrusion was defined as $a \geq 0.2$ $\mathrm{mm}$ mass with an attachment to the luminal surface or floating within the lumen [16]. In the case of ambiguous findings, final diagnosis was determined by consensus of two reviewers. The inter-observer $\mathrm{K}$ coefficient for calcium modification and fracture determined by these reviewers were 0.72 and 0.95 , respectively. The intra-observer $\mathrm{k}$ coefficients for calcium modification and fracture were 0.94 and 0.90 , respectively.

\section{Statistical analysis}


Continuous values are presented as the mean \pm standard deviation with comparisons between the lesions examined by OA and RA evaluated by Mann-Whitney $U$ tests. Categorical variables are presented as a number or frequency, with comparisons performed by the chi-square or Fisher exact test, as appropriate. Cohen's $\mathrm{k}$ was calculated for the intra- and interobserver variability of identification of the calcium modification and fracture by HD-IVUS. With calcium modification and fracture identified by OFDI used as the gold standard, sensitivity, specificity, positive predictive value (PPV) and negative predictive value (NPV) of HD-IVUS were calculated for the purpose of identifying the two findings. $P$ values $<0.05$ were considered statistically significant. All statistical analyses were performed using SPSS 27.0 (SPSS, Inc., Chicago, IL, USA).

\section{Results}

\section{Baseline angiographic and procedural characteristics}

Table 1 presents baseline angiographic and procedural characteristics. Among the 21 heavily calcified coronary lesions in 19 patients (mean age $71.6 \pm 8.2$ years old, male $74 \%$ ), OA and RA were performed in 10 and 11 lesions, respectively. The majority of the target vessels were the left anterior descending artery (67\%), with $86 \%$ of the lesions classified as AHA/ACC type B2/C. For additional plaque modification before stent implantation, cutting or a scoring balloon were used in 16 lesions (76\%). Subsequently, an Ultimaster stent was used in 20 lesions, while an Orsiro stent was implanted in 1 lesion. The mean implanted stent number was $1.5 \pm 0.5$. The mean implanted stent diameter and total implanted stent length were $3.2 \pm 0.4 \mathrm{~mm}$ and $28.0 \pm 6.3 \mathrm{~mm}$, respectively.

\section{Pre-stent and post-PCI OFDI findings}

Table 2 presents the pre-stenting and post-PCI OFDI findings. When focusing on the coronary calcium, the maximum calcium angle was over 270 degrees in most lesions, with a mean \% length of the calcified segment of $85 \%$. Five lesions had a calcified nodule within the stented segment. As a result of the aggressive plaque modification by the atherectomy device along with the pre-dilatation procedures of cutting, scoring or non-compliant balloon, calcium modification and fracture were observed in $15(71 \%)$ and 20 (95\%) lesions, respectively. In addition, medial dissection was observed in 14 (67\%) lesions. With regard to the post-PCI OFDI findings, the average MSA was $5.49 \pm 1.75 \mathrm{~mm}^{2}$ while the average stent expansion was 0.71 . Only 1 lesion had a medial stent edge dissection at the proximal edge. However, this dissection was untreated, as the dissection was located at the ostium of the left descending artery and its flap was short ( $<2 \mathrm{~mm}$ ) and had a narrow-angle ( $<60$ degrees). While all implanted stents were optimized, major acute stent malapposition and in-stent tissue protrusion were observed in 11 lesions (52\%), respectively.

\section{Side-by-side comparison between HD-IVUS and OFDI images}

A total of 1,166 HD-IVUS images were co-registrated with the OFDI images at every 1-mm interval. After exclusion of poor-quality images ( $n=37), 1,129$ OFDI-matched HD-IVUS images were assessed for the 
presence of coronary calcification. A total of 976 (86.4\%) OFDI-matched HD-IVUS images showed coronary calcium. Of these, calcium modification and fracture were found in 201 (20.6\%) and 107 (11.0\%) images, respectively. With OFDI assessment used as the gold standard, sensitivity, specificity, PPV and NPV of HD-IVUS detection for calcium modification and fracture were $54.4 \%, 97.8 \%, 86.7 \%$, $89.1 \%$ and $86.0 \%, 94.5 \%, 58.2 \%, 96.8 \%$, respectively (Figure $2 \mathrm{~A}$ ). When the focus was then placed on the segment with large $(>180$ degrees) calcium $(n=326)$, these values were still similar, with the exception of NPV for the detection of calcium modification and PPV for the detection of calcium fracture (Figure 2B). Figures 3 and 4 show the representative paired images of concordance and discordance cases of calcium modification and fracture. When using HD-IVUS, there were some undetectable calcium modifications observed. These were due to OFDI-derived layered calcium (18.4\%), guide wire artifact (14.9\%) and calcified nodule (4.5\%). In addition, mimicking calcium fracture (pseudo calcium fracture) due to separate calcium was observed in $24.3 \%$ of all of the HD-IVUS-derived calcium fractures. Due to the careful assessment by well-experienced reviewers, all of the guide wire artifacts crossing the coronary calcium, which can mimic calcium fracture, were accurately diagnosed when the HD-IVUS images were reviewed.

\section{Discussion}

\section{Discussion}

This observational study evaluated the diagnostic performance of HD-IVUS of OFDI-derived calcium modification and fracture. The main findings were as follows: 1) As a result of aggressive plaque modification by atherectomy and ballooning, calcium modification and fracture were frequently observed by both OFDI and HD-IVUS. 2) Regarding the detection of calcium modification, HD-IVUS had high specificity, PPV and NPV, while having modest sensitivity. 3) Diagnostic performance of HD-IVUS for the detection of calcium fracture, sensitivity, specificity and NPV was high, although it was only modest for PPV. 4) For the assessment of HD-IVUS images, lesion morphologies and guide wire artifact were potentially related to the misdiagnosis of calcium modification and fracture. These results demonstrate both the capabilities and limitations of HD-IVUS for the treatment of heavily calcified lesions requiring atherectomy.

While IVUS has an excellent detection performance for coronary calcium, it is impossible for IVUS to quantify calcium thickness due to the acoustic shadow $[8,17]$. Therefore, a newly developed reverberation behind post-atherectomy calcium has been considered to be a useful marker of calcium modification [15]. However, our study revealed that more than $40 \%$ of the calcium modifications were undetectable by HD-IVUS. One of the potential reasons for this might be that when using IVUS, the calcified plaque sometimes has signal attenuation behind the coronary calcium. According to pathological reports, when using IVUS, some of the calcified plaques, which include the necrotic core or calcified nodule, potentially have strong signal attenuation $[13,18,19]$. In addition, our study demonstrated that the echo-lucent area behind the layered calcium or calcified nodule did not always have a reverberation echo, despite OFDI confirmation of the calcium modification. Thus, this suggests 
that signal attenuation behind heterogeneous calcium decreases the diagnostic accuracy of HD-IVUS when assessing calcium modification. In other words, HD-IVUS assessment of calcium modification might be useful only for the calcified fibrous plaque. As shown by the representative images, another reason for the discordance of the calcium modification detection is that the guide wire artifact can sometimes hide the narrow-angle reverberation caused by atherectomy. Therefore, when using HD-IVUS to confirm the occurrence of calcium modification, careful reviews around the guide wire artifact will need to be performed.

The present study also showed that the separate calcium determined by HD-IVUS sometimes mimicked a calcium fracture. With regard to the detection of coronary calcium, both IVUS and OFDI had a similar diagnostic performance $[8,20]$. However, when using IVUS, the calcium border is sometimes found to be non-continuous due to non-fibro-dense calcium [19]. Furthermore, since axial resolution of HD-IVUS as compared to OFDI is relatively low $(15-20 \mu \mathrm{m}$ versus $20-40 \mu \mathrm{m})$ [8]. the originally separated calcium and calcium fracture with the small piece of calcium might be difficult to differentiate when using HD-IVUS. Indeed, the PPV of the calcified segments without small ( $\leq 180$ degrees) calcium in the present study was relatively high as compared to the overall calcified segments. Considering the difference in the image reconstruction method and resolution between HD-IVUS and OFDI, it might be unavoidable with regard to making an over- and under-diagnosis of the calcium fracture by HD-IVUS. When performing a quick review of HD-IVUS images in the catheter laboratory, it is potentially easy to make a misdiagnosis for the calcium fracture due to the guide wire artifact that crosses the coronary calcium. If an operator implants a stent in the lesion with thick and large coronary calcium based on this misdiagnosis by the HD-IVUS, this may subsequently be associated with stent underexpansion. In fact, a recent single-center retrospective observational study demonstrated that OFDI-guided RA for calcified coronary lesions resulted in larger stent expansions as compared to the IVUS-guided RA [21]. During HD-IVUS guided PCI for a heavily calcified lesion, it might be important to perform an additive confirmation of full balloon expansion via fluoroscopy to avoid stent underexpansion. In contrast, this study demonstrated that the negative predictive value for detecting the calcium fracture was about $95 \%$, regardless of the size of the coronary calcium. This indicates that HD-IVUS can be used to evaluate ineffective calcified plaque modification.

During the atherectomy procedure, intravascular imaging is helpful for determining the need for additional atherectomy and completion of atherectomy [7]. In particular, as the rotating speed is never displayed during an OA procedure, evaluation of the presence or absence of calcium modification is important for evaluating the efficacy of OA [22]. Therefore, during the OA procedure, OFDI could potentially be very useful for determining the calcium modification. For a heavily calcified lesion, calcium fracture is required to avoid stent underexpansion, and thus, confirmation of the fracture by intravascular imaging could warrant favorable stent expansion $[4,5]$. Recently, intravascular lithotripsy (IVL) has been developed for the treatment of a heavily calcified lesion, thereby making it possible to cause a calcium fracture through the use of shock waves [23]. If IVL becomes the first therapeutic option for the treatment of a heavily calcified lesion, then it is likely that OFDI (or OCT) will be used to a greater extent than HD- 
IVUS due to the high diagnostic accuracy for assessing calcium fracture, as was shown in the present study.

OFDI has an excellent diagnostic performance for calcium modification and fracture. However, it is often unable to obtain an image for severe stenotic lesions due to blood clearance difficulties [8]. Although it is impossible to evaluate the thickness of calcium, IVUS as well as OFDI can determine the degree of the calcium angle [17]. Thus, HD-IVUS is an appropriate imaging modality that can be used to evaluate the need for atherectomy $[7,8]$. Depending on both the situation and the procedural steps, either HD-IVUS or OFDI might be needed for the treatment of a heavily calcified lesion in a single case. To address this, novel hybrid IVUS and OFDI catheter systems have recently been developed by several companies [24]. When these hybrid imaging systems are widely available in daily practice, results of our study will be helpful in determining the effective utilization of these hybrid systems.

\section{Study limitations}

There were several limitations for the present study. First, although more than 1,000 OFDI-matched HDIVUS images were analyzed, the number of enrolled subjects was relatively small. Second, because both the indication and the atherectomy procedure technique vary between physicians or institutes, selection bias could not be avoided. Third, since there was no head-to-head comparison between OFDI and the other HD-IVUS systems, our findings might not be generalizable for the other HD-IVUS systems. Fourth, the clinical impact of the misdiagnosis of calcium modification and fracture on stent expansion and clinical outcomes has yet to be established. A larger clinical study using multi-modalities will need to be conducted to establish the capability of HD-IVUS.

\section{Conclusion}

For a heavily calcified coronary lesion requiring atherectomy, HD-IVUS exhibits a modest diagnostic accuracy for assessing calcium modification and fracture as compared to OFDI. Our study suggests that the use of an OFDI-guided procedure is feasible and recommended in cases with a heavily calcified coronary lesion when these images are available.

\section{Declarations}

\section{Acknowledgement}

This work was supported by KAKENHI Grant Number 20 K08142 from the Japan Society for the Promotion of Science (JSPS).

\section{Compliance with ethical standards}

Conflict of interest Dr. Ishida receives lecture honoraria from Abbott Vascular Japan, Boston Scientific Japan, Daiichi Sankyo, Japan Lifeline, Kowa, Medikit and Terumo Corporation. Dr. Itoh receives lecture 
honoraria from Daiichi Sankyo, and Abbott Vascular. Dr. Morino received lecture honoraria from Abbott Vascular, Boston Scientific, Sanofi, and Daiichi-Sankyo. None of the other authors have any conflicts of interest or financial disclosures.

\section{References}

1. Huisman J, van der Heijden LC, Kok MM, Louwerenburg JH, Danse PW, Jessurun GA, et al. Two-year outcome after treatment of severely calcified lesions with newer-generation drug-eluting stents in acute coronary syndromes: A patient-level pooled analysis from TWENTE and DUTCH PEERS. J Cardiol 2017,69:660-665.

2. Guedeney P, Claessen BE, Mehran R, Mintz GS, Liu M, Sorrentino S, et al. Coronary Calcification and Long-Term Outcomes According to Drug-Eluting Stent Generation. JACC Cardiovasc Interv 2020,13:1417-1428.

3. Hoffmann R, Mintz GS, Popma JJ, Satler LF, Kent KM, Pichard AD, et al. Treatment of calcified coronary lesions with Palmaz-Schatz stents. An intravascular ultrasound study. Eur Heart J 1998,19:1224-1231.

4. Fujino A, Mintz GS, Matsumura M, Lee T, Kim SY, Hoshino M, et al. A new optical coherence tomography-based calcium scoring system to predict stent underexpansion. Eurolntervention 2018,13:e2182-e2189.

5. Fujino A, Mintz GS, Lee T, Hoshino M, Usui E, Kanaji Y, et al. Predictors of Calcium Fracture Derived From Balloon Angioplasty and its Effect on Stent Expansion Assessed by Optical Coherence Tomography. JACC Cardiovasc Interv 2018,11:1015-1017.

6. Raber L, Mintz GS, Koskinas KC, Johnson TW, Holm NR, Onuma Y, et al. Clinical use of intracoronary imaging. Part 1: guidance and optimization of coronary interventions. An expert consensus document of the European Association of Percutaneous Cardiovascular Interventions. Eur Heart J 2018,39:3281-3300.

7. Sakakura K, Ito Y, Shibata Y, Okamura A, Kashima Y, Nakamura S, et al. Clinical expert consensus document on rotational atherectomy from the Japanese association of cardiovascular intervention and therapeutics. Cardiovasc Interv Ther 2021,36:1-18.

8. Maehara A, Matsumura M, Ali ZA, Mintz GS, Stone GW. IVUS-Guided Versus OCT-Guided Coronary Stent Implantation: A Critical Appraisal. JACC Cardiovasc Imaging 2017,10:1487-1503.

9. Chambers JW, Feldman RL, Himmelstein SI, Bhatheja R, Villa AE, Strickman NE, et al. Pivotal trial to evaluate the safety and efficacy of the orbital atherectomy system in treating de novo, severely calcified coronary lesions (ORBIT II). JACC Cardiovasc Interv 2014,7:510-518.

10. Jang IK, Bouma BE, Kang DH, Park SJ, Park SW, Seung KB, et al. Visualization of coronary atherosclerotic plaques in patients using optical coherence tomography: comparison with intravascular ultrasound. J Am Coll Cardiol 2002,39:604-609. 
11. Mintz GS, Douek P, Pichard AD, Kent KM, Satler LF, Popma JJ, et al. Target lesion calcification in coronary artery disease: an intravascular ultrasound study. J Am Coll Cardiol 1992,20:1149-1155.

12. Lee T, Mintz GS, Matsumura M, Zhang W, Cao Y, Usui E, et al. Prevalence, Predictors, and Clinical Presentation of a Calcified Nodule as Assessed by Optical Coherence Tomography. JACC Cardiovasc Imaging 2017,10:883-891.

13. Lee JB, Mintz GS, Lisauskas JB, Biro SG, Pu J, Sum ST, et al. Histopathologic validation of the intravascular ultrasound diagnosis of calcified coronary artery nodules. Am J Cardiol 2011,108:15471551.

14. Yamamoto MH, Maehara A, Karimi Galougahi K, Mintz GS, Parviz Y, Kim SS, et al. Mechanisms of Orbital Versus Rotational Atherectomy Plaque Modification in Severely Calcified Lesions Assessed by Optical Coherence Tomography. JACC Cardiovasc Interv 2017,10:2584-2586.

15. Kim SS, Yamamoto MH, Maehara A, Sidik N, Koyama K, Berry C, et al. Intravascular ultrasound assessment of the effects of rotational atherectomy in calcified coronary artery lesions. Int $\mathrm{J}$ Cardiovasc Imaging 2018,34:1365-1371.

16. Ali ZA, Maehara A, Généreux P, Shlofmitz RA, Fabbiocchi F, Nazif TM, et al. Optical coherence tomography compared with intravascular ultrasound and with angiography to guide coronary stent implantation (ILUMIEN III: OPTIMIZE PCI): a randomised controlled trial. The Lancet 2016,388:26182628.

17. Wang X, Matsumura M, Mintz GS, Lee T, Zhang W, Cao Y, et al. In Vivo Calcium Detection by Comparing Optical Coherence Tomography, Intravascular Ultrasound, and Angiography. JACC Cardiovasc Imaging 2017,10:869-879.

18. Pu J, Mintz GS, Biro S, Lee JB, Sum ST, Madden SP, et al. Insights into echo-attenuated plaques, echolucent plaques, and plaques with spotty calcification: novel findings from comparisons among intravascular ultrasound, near-infrared spectroscopy, and pathological histology in 2,294 human coronary artery segments. J Am Coll Cardiol 2014,63:2220-2233.

19. Ijichi T, Nakazawa G, Torii S, Nakano M, Yoshikawa A, Morino Y, et al. Evaluation of coronary arterial calcification - Ex-vivo assessment by optical frequency domain imaging. Atherosclerosis 2015,243:242-247.

20. Rieber J, Meissner O, Babaryka G, Reim S, Oswald M, Koenig A, et al. Diagnostic accuracy of optical coherence tomography and intravascular ultrasound for the detection and characterization of atherosclerotic plaque composition in ex-vivo coronary specimens: a comparison with histology. Coron Artery Dis 2006,17:425-430.

21. Kobayashi N, Ito Y, Yamawaki M, Araki M, Obokata M, Sakamoto Y, et al. Optical coherence tomography-guided versus intravascular ultrasound-guided rotational atherectomy in patients with calcified coronary lesions. Eurolntervention 2020,16:e313-e321.

22. Shlofmitz E, Jeremias A, Shlofmitz R, Ali ZA. Lesion Preparation with Orbital Atherectomy. Interv Cardiol 2019,14:169-173. 
23. Hill JM, Kereiakes DJ, Shlofmitz RA, Klein AJ, Riley RF, Price MJ, et al. Intravascular Lithotripsy for Treatment of Severely Calcified Coronary Artery Disease. J Am Coll Cardiol 2020,76:2635-2646.

24. Ono M, Kawashima H, Hara H, Gao C, Wang R, Kogame N, et al. Advances in IVUS/OCT and Future Clinical Perspective of Novel Hybrid Catheter System in Coronary Imaging. Front Cardiovasc Med 2020,7:119.

\section{Tables}

Table 1: Baseline patient, angiographic and procedural characteristics 


\begin{tabular}{|c|c|}
\hline Patient number & $\mathrm{n}=19$ \\
\hline Age (years) & $71.6 \pm 8.2$ \\
\hline Male & $14(74)$ \\
\hline \multicolumn{2}{|l|}{ Coronary risk factor } \\
\hline Hypertension & $18(95)$ \\
\hline Dyslipidemia & $13(68)$ \\
\hline Diabetes mellitus & $7(37)$ \\
\hline Current smoking & $4(21)$ \\
\hline Hemodialysis & $1(5)$ \\
\hline \multicolumn{2}{|l|}{ Clinical presentation } \\
\hline Recent myocardial infarction & $2(10)$ \\
\hline Chronic coronary syndrome & $19(90)$ \\
\hline Lesion number & $n=21$ \\
\hline \multicolumn{2}{|l|}{ Angiographic findings } \\
\hline \multicolumn{2}{|l|}{ Culprit vessel } \\
\hline LAD & $14(67)$ \\
\hline LCX & $1(5)$ \\
\hline RCA & $6(29)$ \\
\hline Minimum lumen diameter, mm & $0.59 \pm 0.34$ \\
\hline \% diameter stenosis & $76.3 \pm 14.3$ \\
\hline AHA/ACC type B2/C lesion & $18(86)$ \\
\hline Bifurcation & $9(43)$ \\
\hline \multicolumn{2}{|l|}{ Procedural characteristics } \\
\hline Obtained pre-PCl intravascular imaging & $13(62)$ \\
\hline \multicolumn{2}{|l|}{ Atherectomy device } \\
\hline OA & $10(48)$ \\
\hline RA & $11(52)$ \\
\hline Cutting or scoring balloon usage & $16(76)$ \\
\hline Implanted stent number & $1.5 \pm 0.5$ \\
\hline
\end{tabular}

Page 13/18 


\begin{tabular}{|ll|}
\hline Implanted stent diameter & $3.2 \pm 0.4$ \\
\hline Total implanted stent length & $28.0 \pm 6.3$ \\
\hline
\end{tabular}

Values are $\mathrm{n}(\%)$ or mean \pm standard deviation.

$\mathrm{LAD}=$ left anterior descending artery, $\mathrm{LCX}=$ left circumflex artery, OA = orbital atherectomy, OFDI = optical frequency domain imaging, $\mathrm{PCl}=$ percutaneous coronary intervention, $\mathrm{RA}=$ rotational atherectomy. $\mathrm{RCA}=$ right coronary artery

Table 2: Pre-stenting and post-PCI OFDI findings

\begin{tabular}{|lllll|}
\hline & $\begin{array}{l}\text { Overall } \\
(n=21)\end{array}$ & $\begin{array}{l}\text { OA } \\
(n=10)\end{array}$ & $\begin{array}{l}\text { RA } \\
(n=11)\end{array}$ & $\begin{array}{c}\text { value } \\
\text { Pre-stenting OFDI characteristics }\end{array}$ \\
\hline Maximum calcium angle & $314 \pm 69$ & $335 \pm 55$ & $332 \pm 76$ & 0.18 \\
\hline \% length of calcified segment & $85.0 \pm$ & $84.3 \pm$ & $85.6 \pm$ & 0.61 \\
\hline Presence of calcified nodule & 12.2 & 11.2 & 13.6 & \\
\hline Calcium fracture & $5(24)$ & $1(10)$ & $4(36)$ & 0.16 \\
\hline Calcium modification & $15(71)$ & $9(90)$ & $6(55)$ & 0.07 \\
\hline Medial dissection & $20(95)$ & $10(100)$ & $10(91)$ & 0.33 \\
\hline Post-PCI OFDI characteristics & $14(67)$ & $8(80)$ & $6(55)$ & 0.22 \\
\hline Reference lumen area & & & & \\
\hline Minimum stent area & $7.81 \pm$ & $7.65 \pm$ & $7.96 \pm$ & 0.61 \\
\hline Stent expansion (minimum stent area / reference & 2.50 & 1.76 & 3.11 & \\
\hline lumen area) & $5.49 \pm$ & $5.16 \pm$ & $5.78 \pm$ & 0.51 \\
\hline Major stent edge dissection & 1.75 & 1.54 & 1.94 & \\
\hline Major acute stent malapposition & 0.11 & $0.74 \pm$ & $0.69 \pm$ & 0.25 \\
\hline In-stent tissue protrusion & $1(5)$ & $0(0)$ & $1(9)$ & 0.33 \\
\hline
\end{tabular}

Values are $\mathrm{n}(\%)$ or mean \pm standard deviation. 
$\mathrm{OA}=$ orbital atherectomy, $\mathrm{OFDI}=$ optical frequency domain imaging, $\mathrm{PCl}=$ percutaneous coronary intervention, $\mathrm{RA}=$ rotational atherectomy.

\section{Figures}

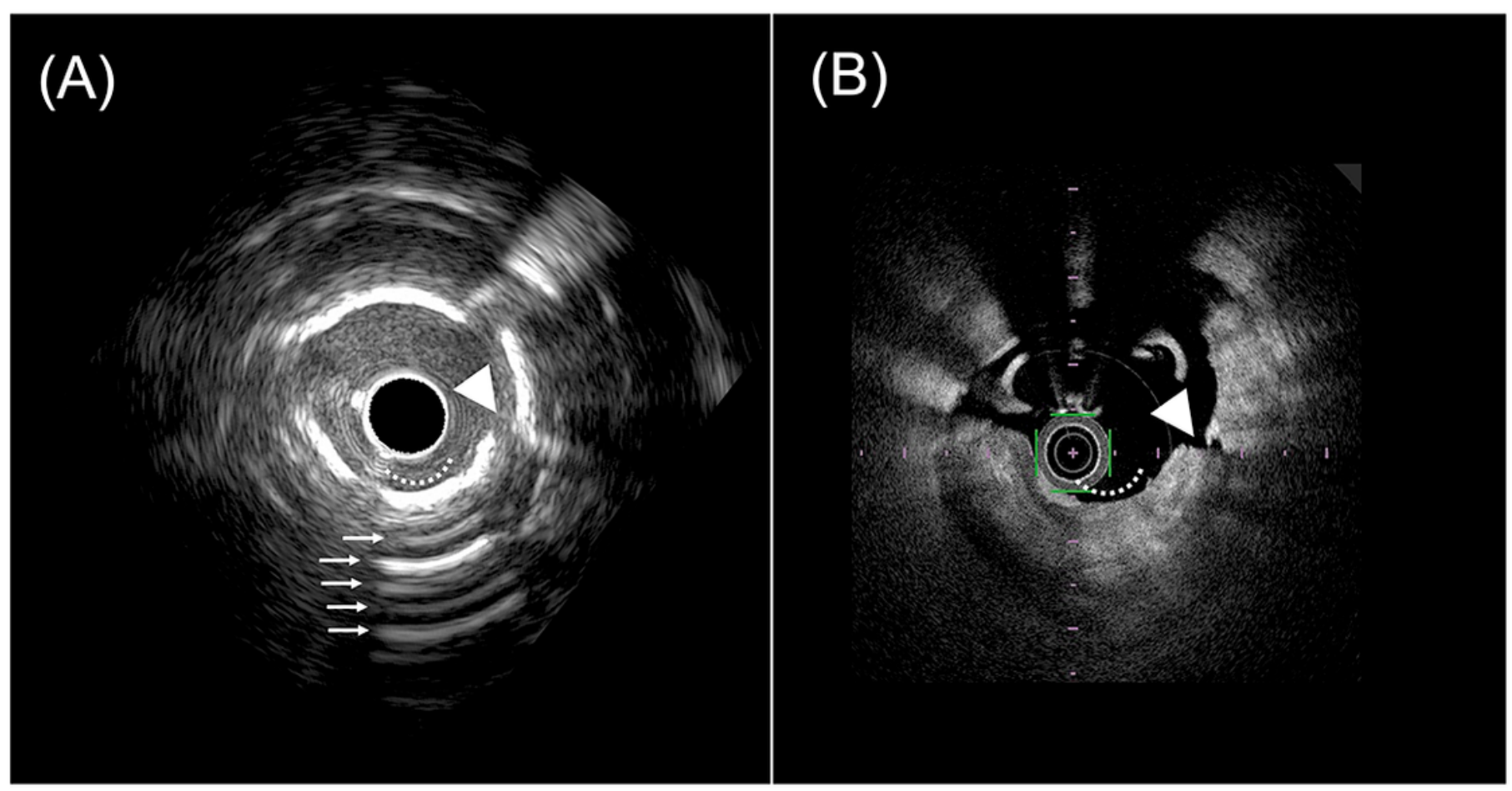

\section{Figure 1}

Definition of calcium modification and fracture. (A) Pre-stenting HD-IVUS image, (B) Pre-stenting OFDI image. $\mathrm{OFDI}=$ optical frequency domain imaging, $\mathrm{HD}$-IVUS $=60 \mathrm{MHz}$ high-definition intravascular ultrasound. Dotted lines = calcium modification, White arrow $=$ reverberation echo, White arrowhead = calcium fracture. Calcium modification was defined as polished and concave-shaped calcium by OFDI and the presence of reverberation with concave-shaped calcium by HD-IVUS. Calcium fracture was defined as the presence of reverberation with concave-shaped calcium. 
(A) Overall

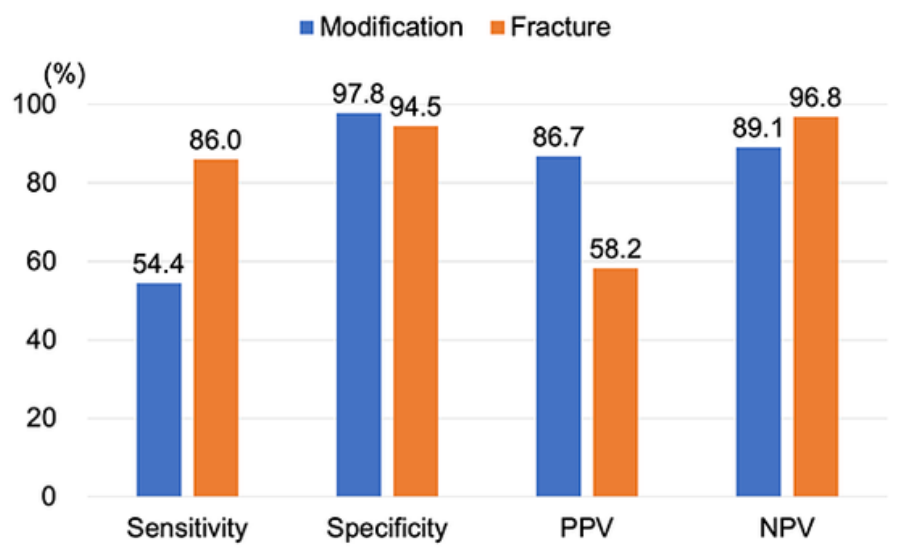

(B) Large $\left(>180^{\circ}\right)$ calcium

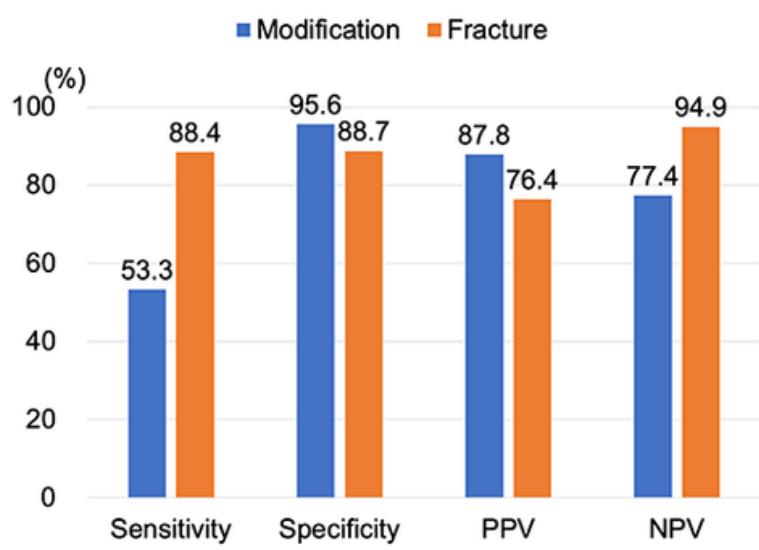

Figure 2

Diagnostic accuracy of HD-IVUS for the detection of calcium modification and fracture. (A) Overall calcified plaque, (B) Large (>180 degrees) calcified plaque. HD-IVUS $=60 \mathrm{MHz}$ high-definition intravascular ultrasound, NPV = negative predictive value, PPV = positive predictive value. 


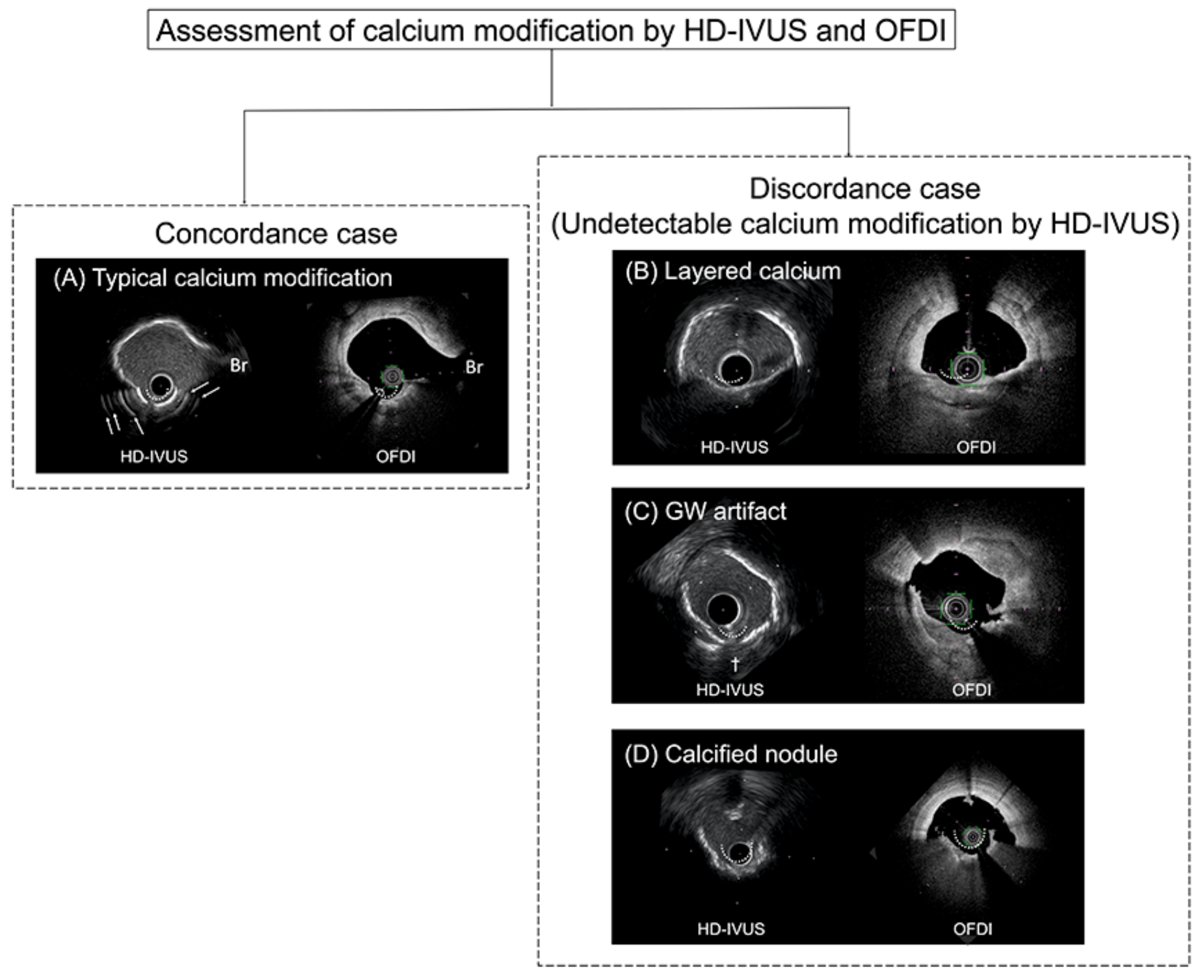

Figure 3

Assessment of calcium modification by HD-IVUS and OFDI. Br = branch of coronary artery, GW = guide wire, OFDI = optical frequency domain imaging, HD-IVUS $=60 \mathrm{MHz}$ high-definition intravascular ultrasound. Dotted lines $=$ calcium modification, White arrow $=$ reverberation echo, $\dagger=\mathrm{GW}$ artifact. 


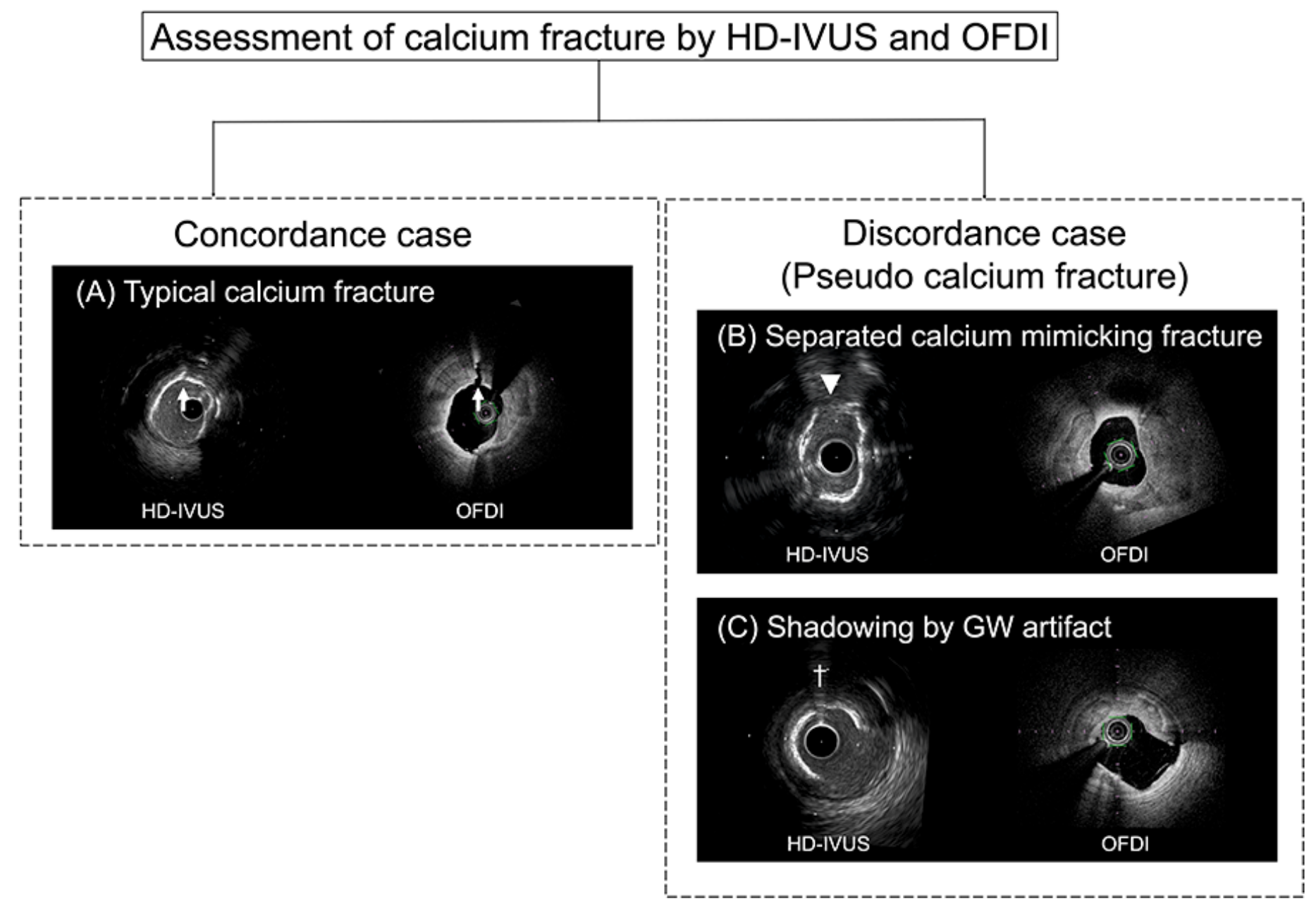

Figure 4

Assessment of calcium fracture by HD-IVUS and OFDI. GW = guide wire, OFDI = optical frequency domain imaging, HD-IVUS = $60 \mathrm{MHz}$ high-definition intravascular ultrasound. White arrow = calcium fracture, White arrowhead $=$ separate calcium mimicking fracture,$\dagger=\mathrm{GW}$ artifact. 